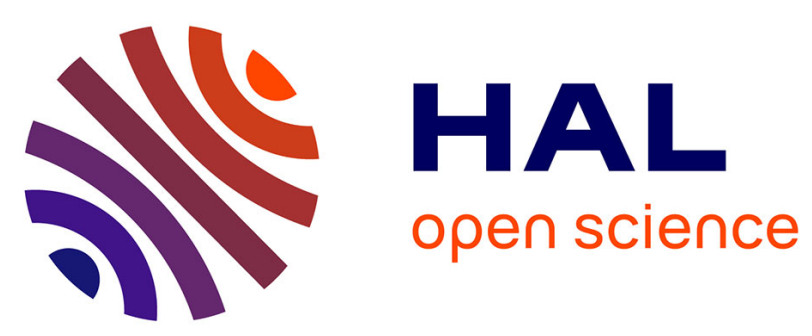

\title{
Characterisation of Knee Brace Migration and Associated Skin Deformation During Flexion by Full-Field Measurements
}

Baptiste Pierrat, Carine Millot, Jérôme Molimard, Laurent Navarro, Paul Calmels, Pascal Edouard, Stéphane Avril

\section{To cite this version:}

Baptiste Pierrat, Carine Millot, Jérôme Molimard, Laurent Navarro, Paul Calmels, et al.. Characterisation of Knee Brace Migration and Associated Skin Deformation During Flexion by Full-Field Measurements. Experimental Mechanics, 2015, 55 (2), pp.349-360. 10.1007/s11340-014-9947-2 . hal01138990

\section{HAL Id: hal-01138990 \\ https://hal.science/hal-01138990}

Submitted on 3 Apr 2015

HAL is a multi-disciplinary open access archive for the deposit and dissemination of scientific research documents, whether they are published or not. The documents may come from teaching and research institutions in France or abroad, or from public or private research centers.
L'archive ouverte pluridisciplinaire HAL, est destinée au dépôt et à la diffusion de documents scientifiques de niveau recherche, publiés ou non, émanant des établissements d'enseignement et de recherche français ou étrangers, des laboratoires publics ou privés. 


\title{
Characterisation of knee brace migration and associated skin deformation during flexion by full-field
}

\section{measurements}

\author{
Baptiste Pierrat · Carine Millot · Jérôme \\ Molimard • Laurent Navarro · Paul \\ Calmels • Pascal Edouard • Stéphane Avril \\ Received: date / Accepted: date
}

\begin{abstract}
Fabric-based knee braces are widely used as orthotic devices to support and align the joint. Despite significant prescription, compliance with the treatment is often negatively affected by discomfort issues, in particular brace slippage and migration. Full-field measurements associated with digital image correlation were performed on 11 subjects to determine if the brace was able to follow skin deformation during knee flexion, which was suspected to be a primary slippage mechanism.

B. Pierrat · C. Millot · J. Molimard · L. Navarro · S. Avril

Ecole Nationale Supérieure des Mines, CIS-EMSE, CNRS:UMR5307, LGF, F-42023 SaintEtienne, France,

E-mail: baptiste.pierrat@gmail.com

B. Pierrat
\end{abstract}

Pôle des Technologies Médicales, F-42000 Saint-Etienne, France

P. Calmels · P. Edouard

Laboratoire de Physiologie de l'Exercice (LPE EA 4338), Université de Saint-Etienne, F-42055

Saint-Etienne, France 
This technique allowed measurement of relatively small slippage magnitudes, which proved to be mostly reversible on re-extension of the leg. Strain fields derived from these measurements indicated a strong influence of gender on skin deformations, and showed that slippage may be due to the inability of the brace fabric to match the non-linear mechanical behaviour of the skin. Consequently, compliance to knee braces may be improved by differentiating their design between genders and judiciously selecting fabrics based on their mechanical behaviour.

Keywords knee braces $\cdot$ compliance $\cdot$ migration $\cdot$ full-field measurements $\cdot$ skin deformation

\section{Introduction}

Knee orthoses, or knee braces are orthotic devices aimed at supporting, aligning, or immobilising the knee joint [8]. They are usually included in the varied methods of treatment and prevention of knee pathologies such as torn ligaments, laxities, arthrosis or general pain. More than 5 million knee braces and supports were sold in the US in 2011 and this market is expected to exceed $\$ 1.2$ billion by 2018 [14]. Despite the fact that they are commonly prescribed by physicians and medical practitioners, their evaluation relies on a small number of biomechanical studies and therapeutic trials $[23,24]$. Their claimed effects are mainly proprioceptive input and joint stabilisation, but their action mechanisms are not fully understood. Orthotic clinicians have reported that the compliance to the orthopædic treatment (i.e. the the degree to which a patient correctly follows the treatment) is often problematic due to discomfort issues, the main cause being brace migration due to slippage $[25,7]$. Although the majority of brace manufacturers state that 
migration is prevented by design features such as silicon pads and a proper fit of the brace by the patient, clinical experience shows that it remains a major issue. This phenomenon is a burden for the patient and also causes a misalignment of the hinge system of the brace, altering lower limb kinematics and potentially leading to abnormal ligament lengths and tensions, as well as other internal mechanical changes $[22,21]$.

Van Leerdam [25] discussed some of the theories behind brace slippage. He listed 4 different causes usually reported by orthotic professionals:

1. Gravity combined with lack of friction control.

2. Tapered shaped legs.

3. Mismatch of anatomical and orthotic centre of rotation.

4. Dynamic forces.

Van Leerdam [25] also proposed a new theory: the main cause could be the inability of the brace to stretch as much as the skin at the anterior side during a knee flexion. This phenomenon is described in Figure 1.

There is a difference between elastic (or reversible) slippage and irreversible slippage. Some elastic slippage may happen during a flexion and be reverted when the leg is stretched back. Despite the fact that this phenomenon is not responsible for brace migration, it makes the brace rub on the skin during each flexionextension and may cause discomfort. Irreversible slippage may also happen during the flexion phase, but the brace does not return to its initial position when the leg is stretched back. The repetition of this phenomenon after multiple flexion-extension cycles causes brace migration. 


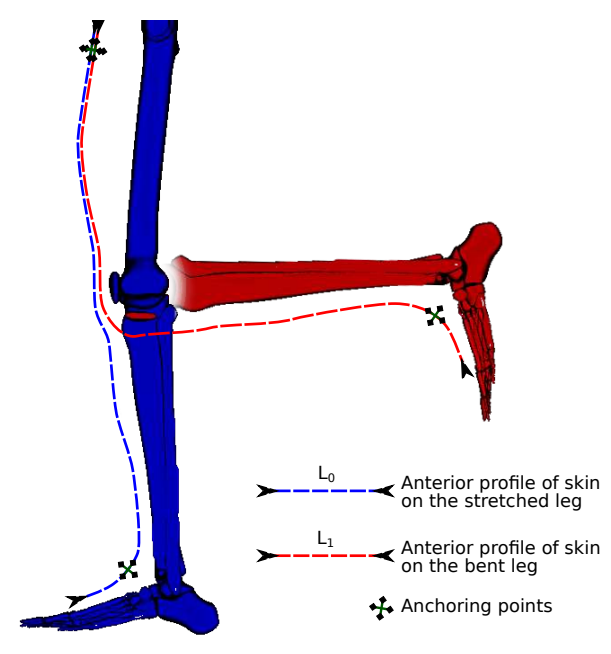

Fig. 1: Knee flexion requires skin stretching. An example is given here between $0^{\circ}$ (blue) and $90^{\circ}$ (red) knee flexion. Under the assumption that skin is able to migrate over underlying tissues [13] and that anchoring points exist at the hip and ankle, the length change along the proximal/distal axis is $\Delta L=L_{1}-L_{0}$. If the brace does not deform as much as the skin, it slips.

The effect of bracing on skin deformation has never been studied and these mechanical phenomena are poorly understood, especially for off-the-shelf fabric hinged braces. This study aims to characterise the ability of such a brace to follow skin deformation during knee flexion in order to understand the mechanisms behind slippage. For this purpose, it was thought that an area where slippage occurs should be observed during a flexion motion. Because the whole brace/skin boundary is covered by fabric hindering measurements, slippage was observed at the brace/skin boundary on the anterior side of the thigh. A full-field measurement technique was used to quantify the migration amplitudes at the brace boundary and the strain fields near this area for a number of subjects. 


\begin{tabular}{|c|c|c|c|c|c|c|c|}
\hline ID & Sex & Age & Size $(\mathrm{m})$ & Weight (kg) & $\begin{array}{c}\text { Thigh circ. } \\
(\mathrm{cm})\end{array}$ & $\begin{array}{c}\text { Skinfold, } \\
\text { extension }(\mathrm{mm})\end{array}$ & $\begin{array}{l}\text { Skinfold, } \\
\text { flexion }(\mathrm{mm})\end{array}$ \\
\hline 1 & $\mathrm{~F}$ & 22 & 1.68 & 53 & 40 & 24 & 25 \\
\hline 2 & $\mathrm{~F}$ & 22 & 1.62 & 52 & 44.5 & 21 & 21 \\
\hline 3 & $\mathrm{~F}$ & 20 & 1.67 & 57 & 45 & 19 & 19 \\
\hline 4 & $\mathrm{~F}$ & 26 & 1.59 & 56 & 47 & 16 & 14 \\
\hline 5 & $\mathrm{~F}$ & 22 & 1.63 & 55 & 48 & 25 & 23 \\
\hline 6 & $\mathrm{~F}$ & 26 & 1.75 & 72 & 51 & 28 & 29 \\
\hline 7 & M & 22 & 1.91 & 80 & 43 & 6 & 5 \\
\hline 8 & M & 22 & 1.79 & 74 & 47 & 15 & 15 \\
\hline 9 & M & 25 & 1.82 & 80 & 48 & 8 & 9 \\
\hline 10 & M & 27 & 1.83 & 73 & 48 & 6 & 7 \\
\hline 11 & M & 22 & 1.81 & 76 & 49.5 & 6 & 7 \\
\hline
\end{tabular}

Table 1: Subject characteristics, ordered by sex and thigh circumference.

\section{Material and methods}

\subsection{Subjects}

A sample of 11 subjects (5 males and 6 females) was selected for this study. These subjects were physically fit, healthy volunteers in their 20s. Their thigh circumference was measured $15 \mathrm{~cm}$ above the knee joint line (found by palpation). Their skinfold was also measured at the anterior side of the thigh, $15 \mathrm{~cm}$ above the knee, using a skinfold calliper in order to estimate subcutaneous fat. Two measures were taken, one at $0^{\circ}$ (extension) and one at $90^{\circ}$ (complete flexion), showing very small differences. The subject characteristics are reported in Table 1. 
1.2 Knee brace

As there is a huge variety of knee orthoses on the market, the focus was placed on manufactured knee braces, as opposed to individualized, custom-made orthotic devices. A simple generic brace using the most common design was used in this study. This design is described in Figure 2. It has been used extensively in previous studies where its mechanical effects have been characterised $[19,20]$. It features a fabric body, bilateral hinged bars and tightening straps. This brace was available in one size fitting a median French male limb (i.e. circumference at the knee: $38 \mathrm{~cm}$, as reported in [15]). Consequently, the brace was purposefully sized incorrectly for all subjects and the effect of this misfitting will be investigated. The absence of patella opening has been shown to have little mechanical effect regarding the stiffening applied by the brace to the joint [20]. This feature is present on many commercial products, but not on this generic design, and might help anchor the brace to the joint and prevent slippage. This is one limitation of this study.

\subsection{Experimental protocol}

The experimental set-up is depicted in Figure 3. In practice, very little information is given to the patients, therefore for the purpose of this study, subjects were asked to fit the brace themselves on their right knee, walk and make adjustments in tightening until they were comfortable. This introduces a source of variability into the study, however it is more representative of real-life scenarios. It was checked that the axis of rotation of the hinged mechanism was aligned with the axis of rotation of the joint. The subjects were seated on a Contrex ${ }^{\circledR}$ MJ (Multi-Joint) system manufactured by CMV AG [9]. This system consists of a dynamometer on 


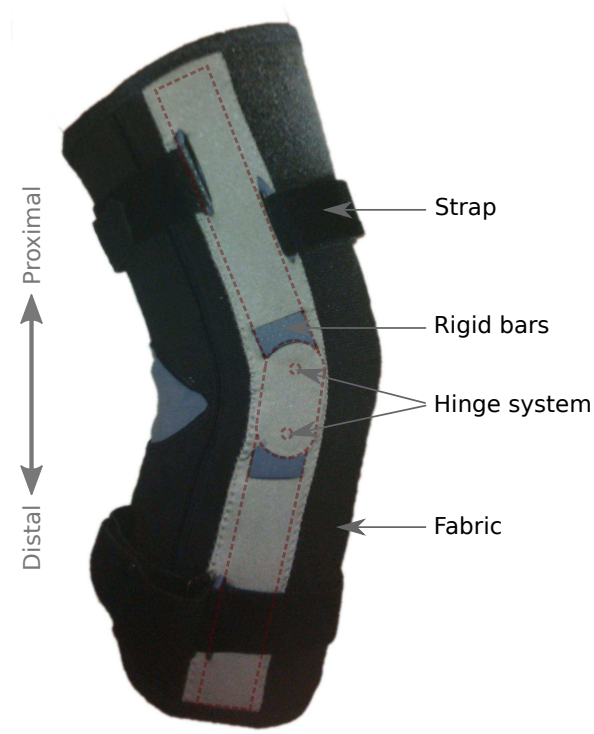

Fig. 2: Generic knee brace with product characteristics.

a swivel arm, it allows a reproducible flexion-extension motion while keeping the leg in a passive state. The volunteers were instructed to keep their leg passive, which will be discussed in Section 3.1 .

A set of three full-field measurements were taken at three different angular positions, before and after 10 complete flexion-extension cycles imposed by the dynamometer at a low speed $(5 \%)$, as described in Table 2 . These respective sets of measurements are referred as "before cycling" and "after cycling".

It is noteworthy that the motion between extension and intermediate position corresponds to typical walking amplitudes, whereas the motion between extension and complete flexion corresponds to typical running amplitudes [18]. For this reason, the two motions will be analyzed separately in order to identify a slipping behavior on both of these types of motion. 


\begin{tabular}{|c|c|c|}
\hline & Knee position & Knee flexion angle \\
\hline \multirow{3}{*}{ 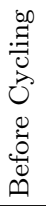 } & Extension & $0^{\circ}$ \\
\hline & Intermediate flexion & $45^{\circ}$ \\
\hline & Complete flexion & $90^{\circ}$ \\
\hline \multirow{3}{*}{ 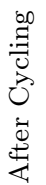 } & Extension & $0^{\circ}$ \\
\hline & Intermediate flexion & $45^{\circ}$ \\
\hline & Complete flexion & $90^{\circ}$ \\
\hline
\end{tabular}

Table 2: Description of the 6 full-field measurements: 3 before and 3 after 10 flexion cycles.

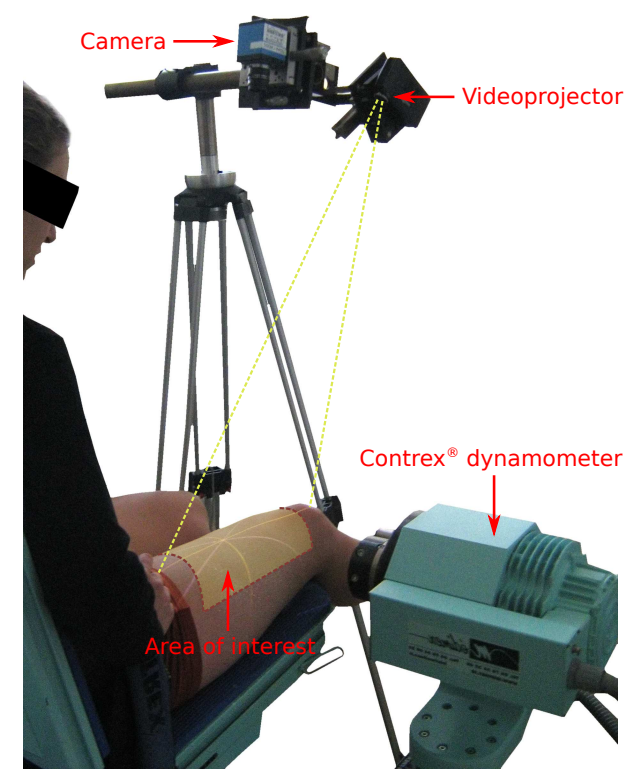

Fig. 3: Experimental set-up (without the brace).

Finally, for each subject, 4 displacement fields were measured: between 0 and $45^{\circ}$ flexion and between 45 and $90^{\circ}$ flexion, before cycling and after cycling. The ROI was a $108 \times 81 \mathrm{~mm}^{2}$ region around the upper boundary of the brace, at the anterior side of the thigh, as shown in Figure 3. 
1.4 Full-field measurement technique

3D displacement fields were estimated using the home-made code Bib_Images ${ }^{\circledR}$ written in Matlab ${ }^{\circledR} 2011$ (The MathWorks, Inc., Natick, Massachusetts, United States) coupling a 2D digital image correlation (DIC) to fringe projection. This method is based on the same optical concepts as stereocorrelation; even if no systematic comparison between the two approaches has been done, it is believed that the high density in shape information brings a better estimate of object slopes, and consequently a better estimate of strain maps. The use of a single camera simplifies the $3 \mathrm{D}$ reconstruction process. These two advantages are balanced by a longer acquisition time, because fringe projection needs a set of images, 11 in this study. This method has been fully described by Molimard et al [17] and a brief summary is given below. The $2 \mathrm{D}$ digital image correlation (DIC) technique was used to compute the in-plane displacements between two states; this displacement is the apparent displacement in the camera's image plane. In conjunction with the 2D DIC, a fringe projection technique, also known as structured light, was used to compute the shape of the region of interest (ROI) before and after the motion. Lastly, a ray tracing technique was used to build the $3 \mathrm{D}$ displacements.

\subsubsection{Frames of reference}

A global and local orthonormal basis systems were used and are defined relative to the undeformed configuration. The global coordinate system $\left(\mathbf{E}_{1}, \mathbf{E}_{2}, \mathbf{E}_{3}\right)$ is defined such that $\mathbf{E}_{1}$ is in the distal direction, $\mathbf{E}_{2}$ in the lateral direction and $\mathbf{E}_{3}$ in the posterior direction, relative to the undeformed configuration. The local coordinate system $\left(\mathbf{B}_{1}, \mathbf{B}_{2}, \mathbf{B}_{3}\right)$ is defined such that: $\mathbf{B}_{3}$ was normal to the surface 
at each point; $\mathbf{B}_{1}$ is the orthogonal projections of $\mathbf{E}_{1}$ on the tangent plane to the surface; $\mathbf{B}_{2}$ is set to complete the orthonormal basis. A representation of these frames of reference is given in Figure 4. Analogous to a cylinder, the local directions $\left(\mathbf{B}_{1}, \mathbf{B}_{2}, \mathbf{B}_{3}\right)$ are called axial, circumferential and normal directions.

\subsubsection{Digital image correlation}

A speckle pattern was applied on the ROI using paint and a toothbrush. Two images $(1280 \times 960$ pixels, $0.084 \mathrm{~mm} /$ pixel $)$ were captured by the camera before and after the motion. Assuming a reference image and a deformed image respectively, with intensity maps $f(r, s)$ and $g(r, s)$ ( $r$ and $s$ being the pixel coordinates), the relationship between $f$ and $g$ is given by:

$$
g(r, s)=f(r+\delta x, s+\delta y)+b(r, s)
$$

where $b$ is the noise.

$(\delta x, \delta y)$ is computed by maximizing the correlation product $\operatorname{corr}(f, g)$. The displacement field is computed at a local scale in the frequency domain by twodimensional discrete Fourier transform on 16 pixels $\times 16$ pixels windows, and subpixel displacement is computed by a spatial phase shifting algorithm for random pattern described in [17]. Measurements were performed at every 5 pixels on a regular grid. Thus, resulting in an undersampled 256 pixels $\times 192$ pixels displacement field.

\subsubsection{Fringe projection}

A periodic pattern of white and black lines was projected on the ROI with an angle $\theta$ between the videoprojector and a recording camera (Figure 3). The defor- 
mation of the fringes, recorded as a phase shift $\varphi(x, y)$, depends on the out-of-plane elevation of the illuminated object $z(x, y)$ :

$$
\varphi(x, y)=\frac{2 \pi \tan (\theta(x, y))}{p(x, y)} z(x, y)
$$

where the pitch $p(x, y)$ is the distance between two light peaks on a flat surface. A calibration procedure is required to get various geometrical parameters. A pine-hole model[6] coupled with camera and videoprojector distortions gives a relationship between the surface point $(x, y, z)$ and the projected one on the CCD plane $(r, s)$ : the position of any point of the ROI in the global coordinate system is known. Coupling the in-plane displacements with the initial and deformed shapes allows for the computation of the displacement $\mathbf{u}$ using the ray tracing procedure.

\subsubsection{Strain derivation}

The pixel position $(r, s)$ makes a convenient curvilinear coordinate system. Knowing the shapes before and after deformation and the displacement field $\mathbf{u}(r, s)$, the deformation gradient tensor $\mathbf{F}$ was computed relative to the global coordinate system:

$$
\mathbf{F}=\mathbf{m}_{i} \otimes \mathbf{M}^{j}
$$

where $\left(\mathbf{m}_{1}, \mathbf{m}_{2}, \mathbf{m}_{3}\right)$ is the covariant basis in the deformed configuration and $\left(\mathbf{M}^{1}, \mathbf{M}^{2}, \mathbf{M}^{3}\right)$ the contravariant basis in the undeformed configuration. The deformed local basis $\left(\mathbf{b}_{1}, \mathbf{b}_{2}, \mathbf{b}_{3}\right)$ was computed from the initial basis $\left(\mathbf{B}_{1}, \mathbf{B}_{2}, \mathbf{B}_{3}\right)$ as:

$$
\mathbf{b}_{i}=\mathbf{F} \mathbf{B}_{i}
$$


The metric tensor is then equivalent to the right Cauchy-Green tensor $\mathbf{C}$, and is calculated as:

$$
\mathbf{C}_{i j}=\mathbf{b}_{i} \cdot \mathbf{b}_{j}
$$

Finally, the in-plane Green-Lagrange strain tensor in the local coordinate system is:

$$
\mathbf{E}=\frac{1}{2}(\mathbf{C}-\mathbf{I})
$$

with $\mathbf{I}$ the identity tensor.

\subsubsection{Post-processing and filtering}

Because of the curvature of the thigh, shape measurements sometimes failed at the edges of the ROI. These areas were manually removed.

As the fringe projection measurements were slightly altered by hair and loose textile fibres, impulse noise was present in the shape results. Using a $2 \mathrm{D}$ discrete convolution, a $3 \times 32$ D gaussian filter (with a standard deviation of 1 ) followed by a same size median filter is applied to the displacement map, and a $72 \mathrm{D}$ median filter and a $132 \mathrm{D}$ median filter were applied to the $x$ - and $y$-shape results, and the $z$-shape results, respectively. The size of the different filters are made small enough to smooth the shape without altering the results. The size of the gaussian and median filters are determined experimentally. The main interest in using these classical filters is the low computing time. 


\subsubsection{Slippage quantification}

Brace slippage results in a displacement discontinuity in the distal direction. Consequently, the slippage magnitude was defined at a point $\left(x_{1}, y_{1}, z_{1}\right)$ as:

$$
\mathbf{u}_{1}\left(x_{1}-a, y_{1}, z_{1}\right)-\mathbf{u}_{1}\left(x_{1}+a, y_{1}, z_{1}\right)
$$

This means that slippage is the difference between adjacent elements of $\mathbf{u}_{1}$ in the distal direction with a distance of $a$. A value of $1.5 \mathrm{~mm}$ was chosen for $a$ based on an adjustment between resolution and noise sensitivity. It was considered that no measurable slippage occurred below $0.2 \mathrm{~mm}$, so the lower threshold was taken at this value.

Both elastic and irreversible slippage may be responsible for the total amount of slippage measured as described. However, by calculating the displacement field between the initial state of the ROI (at $0^{\circ}$ flexion, before cycling) and the same position after cycling, only irreversible slippage remains. Consequently, irreversible slippage was quantified between these two states for each subject using Equation 7.

\subsubsection{Metrological performances}

In a $2 \mathrm{D}$ case, the resolution is quite straightforward, but in a $3 \mathrm{D}$ case, resolution depends on the object shape, and consequently, is more difficult to define. Here, we propose a definition corresponding to a flat surface submitted to in-plane or out-ofplane motions. Then the confidence in the results may be estimated from previous works $[17,16]$. Resolution is estimated using a repeatability test at $5 \mu \mathrm{m}$ in $x$ and $y$, and $50 \mu \mathrm{m}$ in $z$. Strain maps were the most problematic because they are sensitive to small perturbations in displacement fields and shape, and no systematic error evaluation has been performed. Gradient estimation procedures were based on 
previous well-known works [4] (displacement gradients were obtain using a diffused approximation approach [2]) and strain calculation has been tested on numerical cases (no systematic error has been found on simple cylindrical cases). In the following section, results were analysed away from the borders of the ROI and from discontinuities at the boundary, and average values were extracted. However, the resolution of the full-field measurement system was high enough to measure small magnitudes of slippage, and strain resolution was estimated to be $4 \times 10^{-4}$. Spatial resolution is defined according to Badulescu et al [3]. Its determination is calculated from the autocorrelation function of pure noise obtained with a repeatability test though the extraction procedure [4]. In this case, its value corresponds to 1 pixel i.e. $0.084 \mathrm{~mm}$ for the shape, and $0.66 \mathrm{~mm}$ for the displacements. Strain resolution is more difficult to characterize, as already mentioned by Berge-Gras and Molimard [4]; a reasonable value in the gradient direction is $1.0 \mathrm{~mm}$. Values for resolution and spatial resolution are given without post-processing (filtering), except in the case of strain maps.

\section{Results}

Shapes and displacements were successfully measured using the described methods. Despite the high displacement magnitudes (in the order of 1-2 cm) occurring within the ROI, the combined DIC and fringe project methods performed appropriately. This chapter will describe the typical experimental results on a single subject in detail which is then followed by the pooled results of all volunteers. Finally, further experiments on elastic/inelastic slippage were performed on a single subject and will be presented as a case study. 


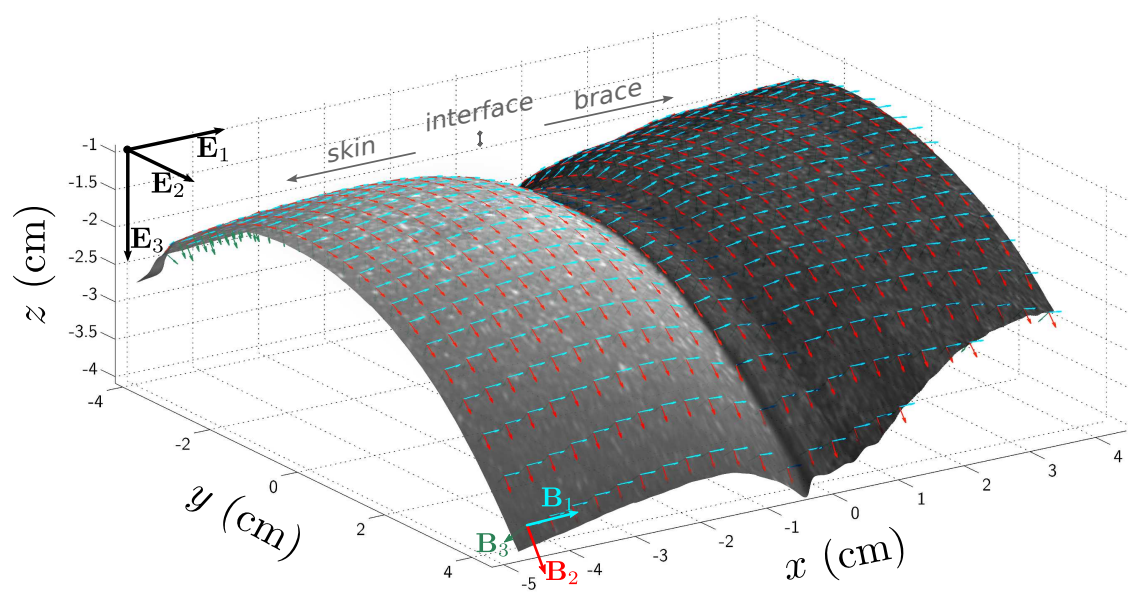

Fig. 4: Representation of the shape of the ROI in the initial configuration (stretched leg). The global coordinate system $\left(\mathbf{E}_{1}, \mathbf{E}_{2}, \mathbf{E}_{3}\right)$ is represented as well as the local coordinate system $\left(\mathbf{B}_{1}, \mathbf{B}_{2}, \mathbf{B}_{3}\right)$ at each point. The reader is reminded that direction $\mathbf{E}_{1}$ is the distal direction (towards the knee).

\subsection{Typical case}

The results presented for this case are before cycling. The shape as measured by fringe projection in the initial state is depicted in Figure 4. Filtering was effective at removing impulse noise while preserving the shape discontinuity at the boundary. At the extremity of the brace, near the boundary, it can be noticed that the brace featured an elastic band that had the effect of compressing the thigh.

Cross-sections at $y=0$ were plotted for the three positions $\left(0^{\circ}-45^{\circ}-90^{\circ}\right.$ flexion) and were reported in Figure 5. Here it can be noticed that the skin is bulging upward ( $z$ direction) near the boundary due to the compression of soft tissues against the brace at $0^{\circ}$ flexion. Between $0^{\circ}$ and $45^{\circ}$, the boundary migrated of about $1.5 \mathrm{~cm}$ to the right towards the knee. This had the effect of stretching the skin and underlying soft tissues, and the soft tissues retracted in the $z$ direction. 


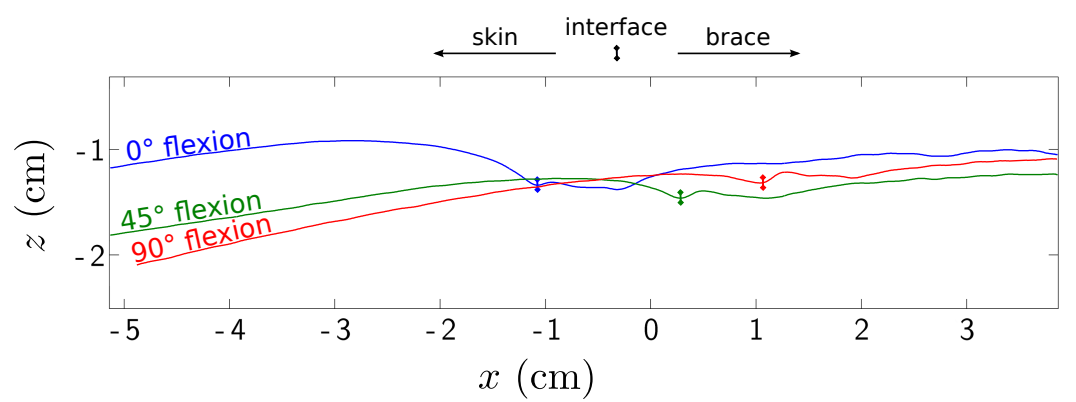

Fig. 5: Cross-sections at $y=0$ showing the shape profile for the three positions $\left(0^{\circ}-45^{\circ}-90^{\circ}\right.$ flexion). The skin is on the left side, the brace on the right side and the boundary is represented by a marker.

The bulging was relieved as the fabric is stretched away from the brace boundary. Between $45^{\circ}$ and $90^{\circ}$, the boundary continued to migrate $0.8 \mathrm{~cm}$ towards the knee but soft tissues did not retract as much in the $z$ direction.

Displacement and strain maps are reported in Figure 6. They showed the highest magnitudes between $0^{\circ}$ and $45^{\circ}$, as expected from Figure 5 . The skin and brace migrated between 1 and $1.6 \mathrm{~cm}$ in the distal direction $\left(\mathbf{u}_{1}\right)$. The magnitude was slightly higher in the central area, at the most anterior part of the thigh. The displacement $\mathbf{u}_{2}$ in the lateral direction was smaller, showing that the migration is almost unidirectional. However this migration was also accompanied by a flattening of the thigh, as shown by the posterior displacement $\mathbf{u}_{3}$ : the most anterior part moved posteriorly while the sides of the thigh moved slightly anteriorly. Regions of interest were defined on the skin and the brace; the average displacement of these areas characterized the migration of each part.

In order to compute strain maps, the shape and displacements have to be derived numerically. This was problematic around discontinuities or at places with small measurement inaccuracy, as these inaccuracies become amplified. Problem- 

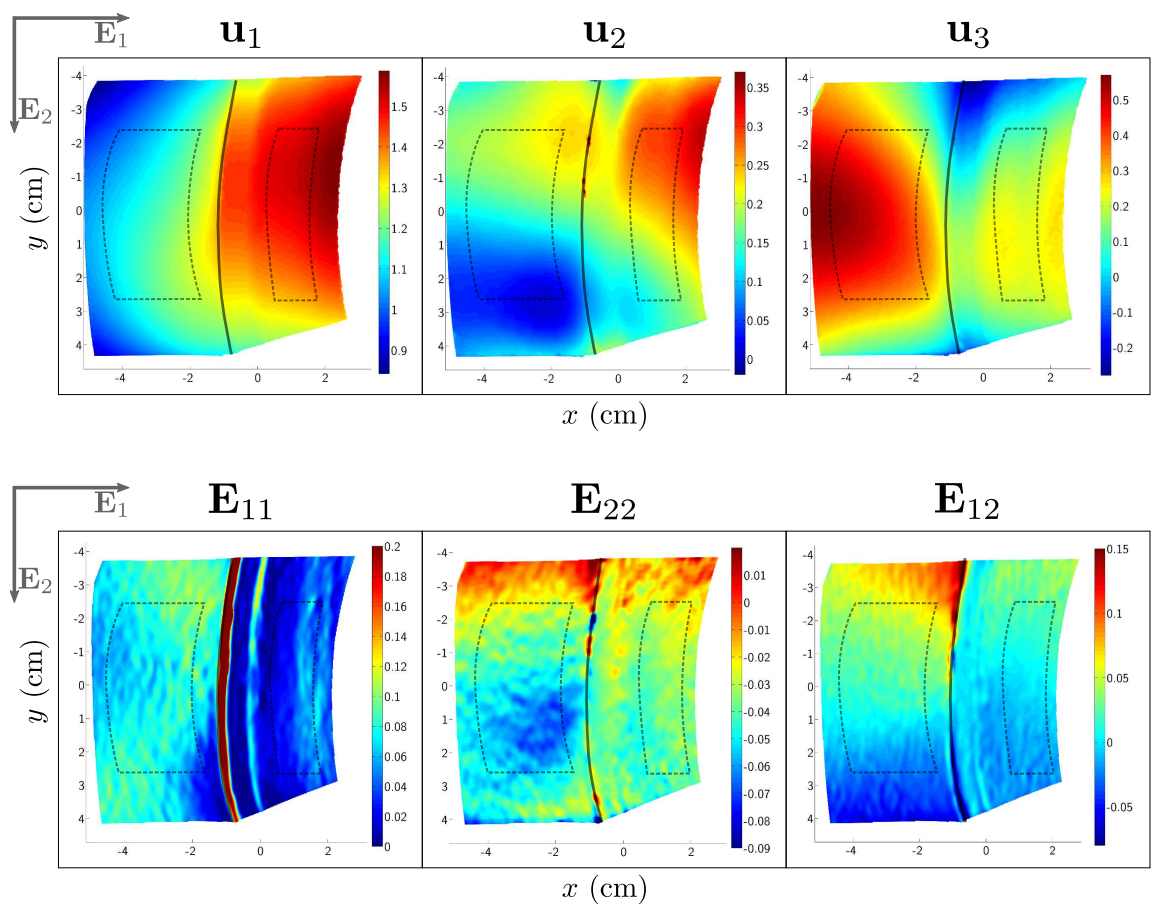

Fig. 6: Displacement $\mathbf{u}$ in the global frame of reference (unit: $\mathrm{cm}$ ) and in-plane Green-Lagrange strain $\mathbf{E}$ in the local frame of reference, between $0^{\circ}$ and $45^{\circ}$ flexion. The skin/brace boundary is represented by a solid line and the regions of interest of the skin and brace by dashed lines. Note that the high strain band in $\mathbf{E}_{11}$ is an artefact due to slippage.

atic areas were the edges of the ROI, the brace/skin boundary and the elastic band area on the brace. Outside these areas, in regions of interest represented in Figure 6 , strain fields were rather homogeneous and an average strain was computed in both windows. By looking at the strain fields (especially $\mathbf{E}_{11}$ ), it is possible to distinguish the different areas of the ROI: skin (high deformation), elastic band (undetectable deformation) and brace fabric (low deformation). In this case, the average strain in the axial direction was $7.51 \%$ in the skin and $3.28 \%$ in the brace. Respective values in the circumferential direction were $-4.49 \%$ and $-3.39 \%$, and $1.85 \%$ and $0.38 \%$ in the shear direction. 


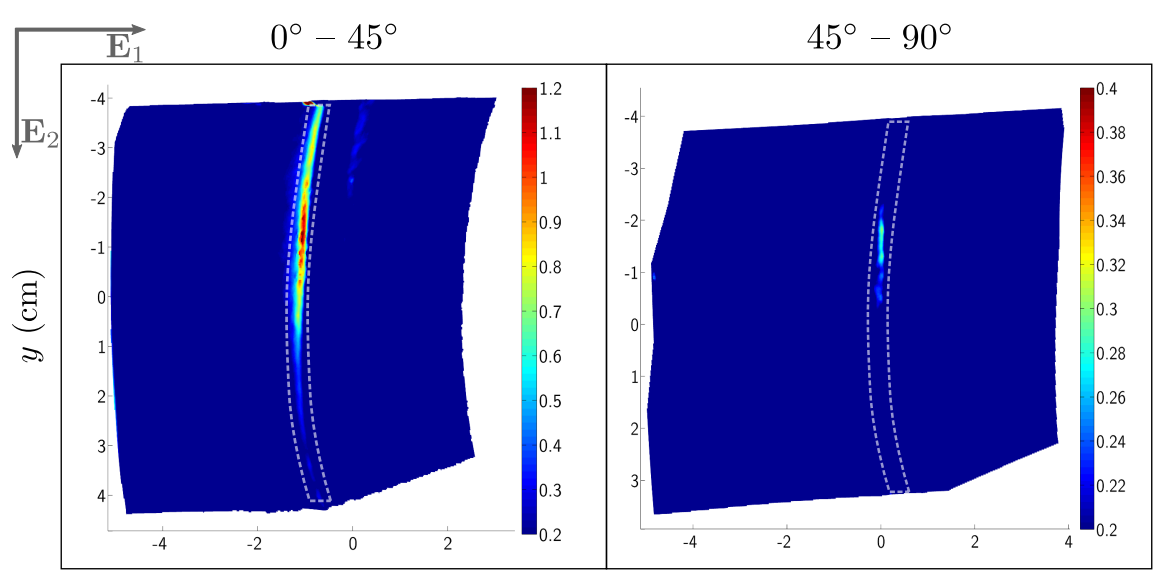

$x(\mathrm{~cm})$

Fig. 7: Slippage magnitude (in $\mathrm{mm}$ ) as defined in Section 1.4.6 for the two steps of the motion. Boundaries are represented by dashed lines.

Looking at displacement $\mathbf{u}_{1}$, a discontinuity can be observed at the boundary. This translates as brace slippage between position 1 and 2 . The slippage magnitude (both elastic and irreversible, as defined in Section 1.4.6) of this case has been represented in Figure 7. During the first half of the motion, slippage occurred over the whole boundary, with magnitudes up to $1.2 \mathrm{~mm}$, whereas the second half of the motion only involved some localized slippage of magnitude $0.3 \mathrm{~mm}$. It can be noticed that the magnitude is maximal in the centre area and minimal on the sides, meaning that the brace was pulled more strongly at the anterior part of the limb and remained in place on the medial and lateral sides, where the hinged bars are located.

\subsection{Pooled results}

The data processing presented in Section 2.1 was performed for the 11 subjects, between $0^{\circ}-45^{\circ}$ and $45^{\circ}-90^{\circ}$, and before/after cycling. 
On average, for all the subjects, the first motion was responsible for the majority of displacement, strain and slippage. However, a difference in mechanical behaviour may be noticed between the skin and brace. The skin largely deformed during the first flexion $(8.59 \%)$ and much less during the second $(2.25 \%)$, while the brace deformation was roughly the same $(2.84 \%-2.52 \%)$. This was assumed to be caused by the highly nonlinear behaviour of skin [12], whereas mechanical characterisation of the brace fabric showed a linear behaviour up to about $30 \%$ strain [19]. Mean values of total circumferential and shear strain in the skin were $(-5.25 \pm 2.08) \%$ and $(2.22 \pm 2.19) \%$ respectively.

The variation in skin and brace migration was small, indicating that these quantities depend on the motion and were not specific to a single subject, nor to the positioning of the brace which was fitted by the subject themselves. On the other side, the variation in strain was much larger.

Interesting results are shown by separating male and female subjects and are reported in Table 3. There was a significant difference in skin strain between females and males: total average values were $(15.0 \pm 1.34) \%$ and $(5.90 \pm 0.60) \%$ respectively. This difference is not due to morphological differences as thigh circumferences were not significantly different between gender. This difference was also observed for the total slippage: average values were $(1.87 \pm 0.30) \%$ (females) and $(0.89 \pm 0.32) \%$ (males). No significant correlation was found between morphological/physiological quantities (height, weight, skinfold, thigh circumference) and variables described in Table 3 . It is particularly interesting to note the absence of correlation between thigh circumference and slippage magnitude, showing that morphology was not the primary factor inducing slippage here. 


\begin{tabular}{|c|c|c|c|c|}
\hline \multirow{6}{*}{$\begin{array}{l}\stackrel{i n}{\rightarrow+} \\
1 \\
:\end{array}$} & & Females (av. \pm st. dev.) & Males (av. \pm st. dev.) & $p<0.05$ \\
\hline & Skin migration $(\mathrm{mm})$ & $13.9 \pm 2.27$ & $17.1 \pm 2.86$ & 夫 \\
\hline & Brace migration $(\mathrm{mm})$ & $17.8 \pm 2.48$ & $19.4 \pm 2.59$ & \\
\hline & $\mathbf{E}_{11}$ of skin (\%) & $11.3 \pm 2.04$ & $5.37 \pm 1.04$ & $\star$ \\
\hline & $\mathbf{E}_{11}$ of brace $(\%)$ & $2.61 \pm 0.72$ & $3.11 \pm 1.81$ & \\
\hline & Max. slippage (mm) & $1.50 \pm 0.25$ & $0.72 \pm 0.21$ & $\star$ \\
\hline \multirow{5}{*}{$\begin{array}{l}\stackrel{8}{8} \\
1 \\
1 \\
\text { is }\end{array}$} & Skin migration $(\mathrm{mm})$ & $6.23 \pm 0.78$ & $6.98 \pm 0.63$ & \\
\hline & Brace migration $(\mathrm{mm})$ & $7.85 \pm 0.90$ & $7.66 \pm 0.55$ & \\
\hline & $\mathbf{E}_{11}$ of skin (\%) & $3.69 \pm 1.17$ & $0.53 \pm 0.66$ & $\star$ \\
\hline & $\mathbf{E}_{11}$ of brace (\%) & $2.10 \pm 0.62$ & $3.02 \pm 1.08$ & \\
\hline & Max. slippage $(\mathrm{mm})$ & $0.36 \pm 0.14$ & $0.17 \pm 0.13$ & $\star$ \\
\hline \multirow{5}{*}{ 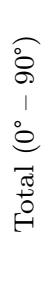 } & Skin migration $(\mathrm{mm})$ & $20.1 \pm 1.85$ & $24.0 \pm 2.56$ & $\star$ \\
\hline & Brace migration $(\mathrm{mm})$ & $25.7 \pm 1.78$ & $27.0 \pm 2.42$ & \\
\hline & $\mathbf{E}_{11}$ of skin (\%) & $15.0 \pm 1.32$ & $5.91 \pm 0.60$ & 夫 \\
\hline & $\mathbf{E}_{11}$ of brace $(\%)$ & $4.70 \pm 1.11$ & $6.14 \pm 2.61$ & \\
\hline & Max. slippage (mm) & $1.87 \pm 0.30$ & $0.89 \pm 0.32$ & $\star$ \\
\hline
\end{tabular}

Table 3: Results for female and male subjects, before cycling: migrations and axial strain of skin and brace in the respective regions of interest and maximum slippage at the boundary. Lines with a star mean a $p$-value $<0.05$ (given by a homoscedastic T-test between the two genders).

In order to determine the cycling effect, data was processed the same way after 10 flexion cycles. This cycling effect was defined as the ratio between a quantity before cycling to the quantity after cycling. The obtained values are reported in Table 4. Cycling had no significant effect on skin and brace migration. However, it slightly increased total strain, especially in the skin, as well as the average slippage magnitude. The viscoelastic nature of the skin had a significant 


\begin{tabular}{lccc}
\hline & $0^{\circ}-45^{\circ}$ & $45^{\circ}-90^{\circ}$ & Total $\left(0^{\circ}-90^{\circ}\right)$ \\
\cline { 2 - 4 } Skin migration & $1.05 \pm 0.16$ & $0.93 \pm 0.12$ & $1.01 \pm 0.11$ \\
Brace migration & $1.09 \pm 0.14$ & $0.91 \pm 0.13$ & $1.03 \pm 0.09$ \\
$\mathbf{E}_{11}$ of skin & $1.32 \pm 0.24$ & $0.60 \pm 0.79$ & $1.19 \pm 0.17$ \\
$\mathbf{E}_{11}$ of brace & $1.01 \pm 0.35$ & $0.82 \pm 0.32$ & $0.91 \pm 0.18$ \\
Av. slippage & $1.23 \pm 0.16$ & $1.02 \pm 0.85$ & $1.14 \pm 0.14$ \\
\hline
\end{tabular}

Table 4: Cycling effect (defined as the ratio between a quantity before cycling to the quantity after 10 flexion cycles) on average values of different measured quantities.

effect on the total strain: skin tends to get softer after stretching cycles. Another interesting phenomenon is the difference observed between the two flexion steps. Cycling caused a $32 \%$ increase in strain in the first part of the flexion phase, and a decrease of this variable in the second part.

Finally, the irreversible slippage caused by the 10 cycles was determined for all patients, as described in Section 1.4.6. An average value of $(0.21 \pm 0.14) \mathrm{mm}$ was found. This amount is very low and indicates that most slippage is elastic.

\subsection{Case study}

The previous results were very interesting but somewhat limited to elastic slippage, so further experiments were performed on a single female subject. The following results should therefore be taken with caution as they may not be representative of the whole population.

As the brace was suspected to have an effect on skin strain, skin strain was measured without the brace and compared to results obtained with the brace worm by the patient. These fields are depicted in Figure 8. It is clear that bracing 


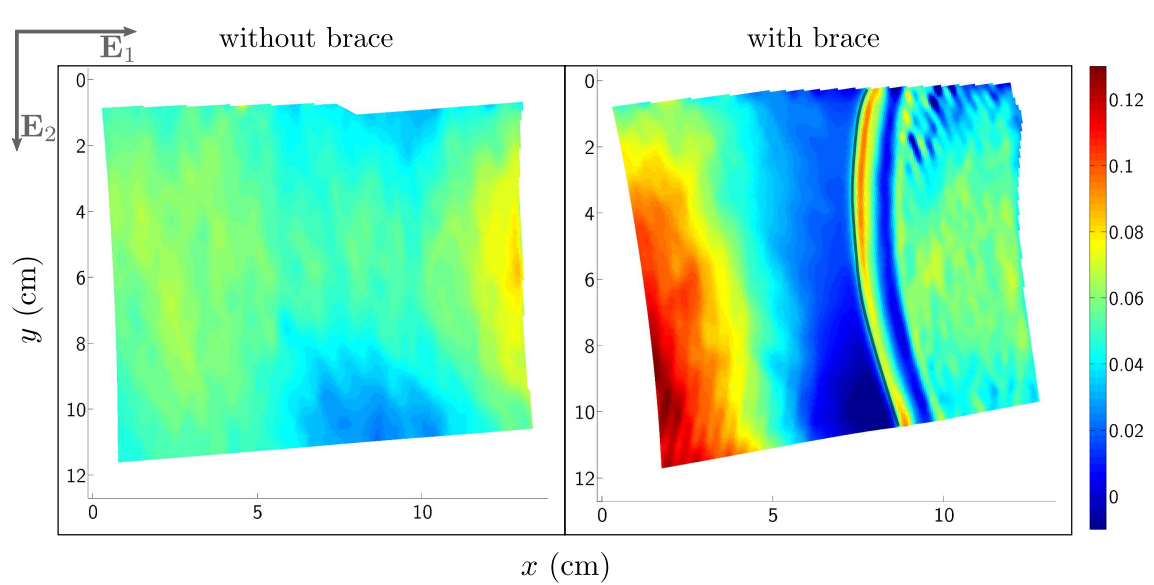

Fig. 8: Longitudinal strain $\left(\mathbf{E}_{11}\right)$ in the ROI without/with the brace.

highly increased longitudinal strain in the skin: values increased from $6 \%$ up to $10-12 \%$ in the left part. This phenomenon is likely due to the brace pulling on the skin during the flexion phase and might be caused by the inability of the brace to stretch enough to fit normal skin deformation in the joint area (as described in Figure 1), leading to a pulling of proximal skin to compensate. This pulling was confirmed by measures of distal displacements, which was much higher with the brace than without $(2.5 \mathrm{~cm}$ and $1.2 \mathrm{~cm}$ in the centre of the ROI respectively).

On the same subject, 20 complete flexion-extension cycles were performed and measurements were taken at $0^{\circ}$ flexion and $90^{\circ}$ flexion during cycles number 1,5 , $10,15,20$. It was then possible to determine:

1. Elastic slippage between $0^{\circ}$ and $90^{\circ}$, as done previously, and for each step.

2. Strains between $0^{\circ}$ and $90^{\circ}$, as done previously, and for each step.

3. Inelastic slippage relative to the first position after $N$ cycles by computing displacements between the following states:

(a) Reference state: initial position, at $0^{\circ}$ flexion before the first motion. 


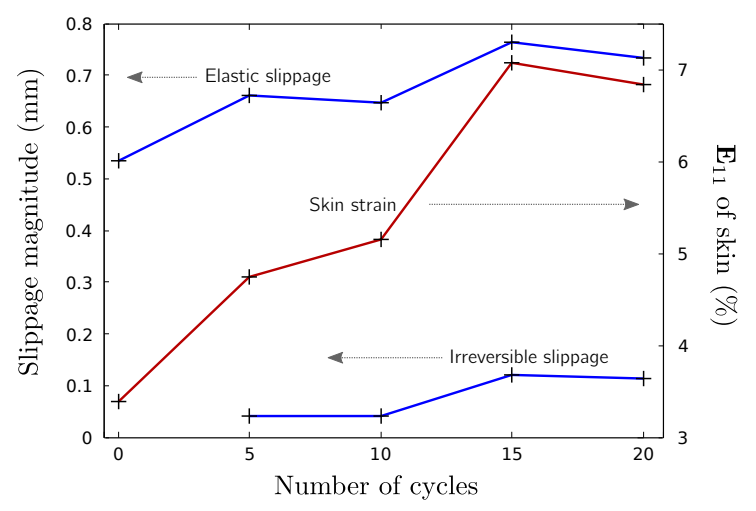

Fig. 9: Elastic slippage between $0^{\circ}$ and $90^{\circ}$ (left scale), irreversible slippage relative to the initial state after $N$ cycles (left scale) and evolution of the longitudinal skin strain between $0^{\circ}$ and $90^{\circ}$ as a function of the number of cycles (right scale).

(b) $N$ th deformed state: same position $\left(0^{\circ}\right.$ flexion) but after having performed $N$ th cycles.

Results are presented in Figure 9. Elastic slippage between the straight and bent leg slightly increased from 0.54 to $0.73 \mathrm{~mm}$ after 20 cycles. Skin strain in the axial direction showed a high increase: during the first flexion motion, skin deformed by $3.5 \%$, and this magnitude increased by a factor 2 after 15 and 20 cycles, highlighting the high visco-elasticity of skin. Finally, negligible amounts of irreversible slippage were observed in this case, slightly below the no-slippage threshold.

\section{Discussion}

3.1 Methodology and analysis of the results

Cycling was limited to 10 cycles for the general study, which is a small amount compared to the number of flexion cycles performed during a day. However, this 
study focused only on the slippage mechanism consisting of the inability of the brace to stretch as much as the skin at the anterior side. In this sense, the case study on one subject showed only elastic slippage, and no significant irreversible migration. Consequently, the slippage phenomenon described in Figure 1 (inability of the brace to stretch as much as the skin) was not conclusively reproduced in this case. However, results of the case study tend to show that elastic slippage increases with the number of cycles. More cycles might lead to irreversible migration, for example due to increased skin lubrication (sweating). The number of cycles required to observe conclusive irreversible slippage might not have been reached.

As the subjects were seated, the posterior part of the fabric was slightly compressed between the thigh and the seat. This was not problematic as the studied slippage mechanism is responsible for slippage at the anterior part of the leg. Nevertheless, soft tissues were also not exactly shaped the same way they are in a standing position, but this was assumed to have a negligible effect on the results.

Van Leerdam [25] measured skin migration on one subject for an approximate flexion angle of $90^{\circ}$ with marks drawn on the skin, and obtained roughly $4 \mathrm{~cm}$. As their area of interest was closer to the knee, their result is consistent with the presented values. Brownstein [7] measured brace migration of 14 knee braces after 15 min of exercise and found magnitudes from 0.25 to $11 \mathrm{~mm}$, with an average of $4 \mathrm{~mm}$. As other slippage mechanisms were involved and brace models were very different (functional rigid hinged braces from the U.S. market), it is difficult to compare these results to the present study. Bethke [5] placed 156 markers at the surface of the leg and measured strain fields during a $90^{\circ}$ flexion; longitudinal strains of $30 \%$ were found $6 \mathrm{~cm}$ above the patella, which is a little higher than values measured in this study (11\% on average). However, the area of interest was 
more proximal in the present study, and it is suspected that strains are high in the patella area and rapidly decrease away from this region (as seen in Figure 8).

The difference in strain magnitudes between males and females has already been reported in terms of mechanical properties by Diridollou et al [11], but other studies $[1,10]$ found no statistical difference between genders. As the measured average strains are so different, it is highly probable that a mechanical or anatomical difference exists between young males and females.

As stated previously, the first half of the flexion phase reproduced walking conditions while the entire motion was more similar to running amplitudes. As the majority of migration, deformation and slippage occurred between $0^{\circ}-45^{\circ}$, braces suited for walking amplitudes should still adhere during static motions with higher amplitudes. Note that dynamic forces, which were not investigated, may become a primary mechanism is the dynamic case (ex: running), and that viscoelastic effects may also come to play. Also, a complete passive state of the muscles is not expected during normal use, and this could also influence the results. However, there is no evidence that muscle contraction induces slippage, as it had not been reported as a slipping mechanism by Van Leerdam [25].

\subsection{Mechanical analysis}

Despite the fact that no irreversible migration was found, the presented methods did provide insight into the possible mechanism underlying migration. The effect of bracing on skin deformation showed that if the brace fabric is not able to deform as much as the skin in the longitudinal direction, two mechanisms are in competition: slippage and skin pulling. Even if such results were shown for only one subject, it 
is likely that this behaviour is reproducible. If the ability of the brace to deform is exceeded by a large longitudinal deformation of the skin, slippage should occur in a stick-and-slip fashion.

Even if it would be preferable to prevent slippage, it would mean that skin deformation underneath the brace should be kept at the same magnitude as the brace deformation, by increasing the adherence of the device to the skin; consequently, it would also mean larger strains for the skin in regions around the brace. However, both mechanisms may cause discomfort issues and should be avoided if possible.

The tensile behaviour of skin and brace fabric is depicted in Figure 10. Although properties of skin vary widely among individuals, the difference in terms of mechanical behaviour is obvious: skin stiffness is very soft at low strains, then its stiffness rises rapidly while the brace fabric curve is slightly convex and more linear. This explains why skin deformed much more than the brace during the first half of the flexion phase (Table 3); the fact that brace stiffness is much higher than skin stiffness for strains below $20 \%$ is likely to be the main cause for brace slippage or skin pulling in these conditions.

\subsection{Clinical and manufacturing outcomes}

The presented results and interpretations may be of interest to clinicians and orthotic manufacturers for the benefit of patients.

It has been shown that a major issue of these fabric-based braces is the difference in terms of mechanical behaviour between fabric in the longitudinal direction and the underlying skin (Figure 10). On such braces, circumferential stiffness will 


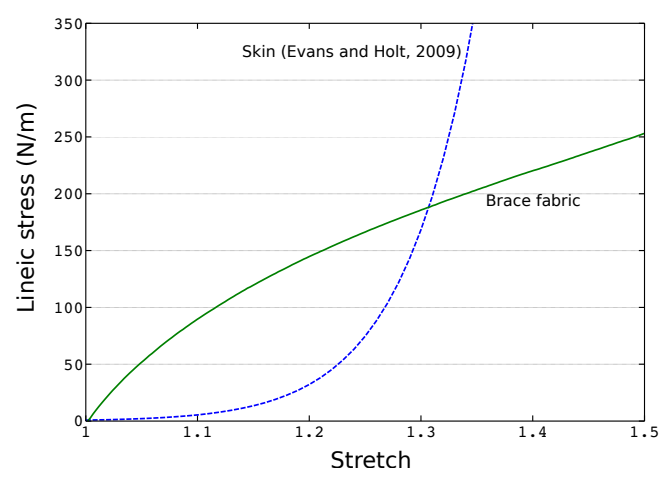

Fig. 10: Lineic stress (force divided by sample width) vs. stretch for skin and brace fabric (longitudinal direction) under unidirectional tension. Skin behaviour was plotted using an Ogden constitutive model and the identified parameters from Evans and Holt [12] with a initial strain of 0.2 and a thickness of $1 \mathrm{~mm}$. Brace fabric was identified as previously described [19].

be responsible for the compressive effect and maintaining the rigid bars; as shown, the fabric does not deform substantially in this direction during flexion. As for the longitudinal direction, large deformations occur during flexion. Consequently, it might be advisable to select warp and weft threads with different mechanical behaviours, in such a way that each direction would be optimized for its corresponding mechanical role. A brace with a longitudinal mechanical behaviour similar to the skin's would be more comfortable: slippage due to skin migration and stretching would be reduced as well as distal skin pulling, because it would be able to deform together with the lower limb surface.

As the difference in skin mechanical behaviour was very important between genders, it might be valuable to translate this difference in terms of brace design, once again by optimizing the longitudinal mechanical behaviour of the fabric. However, further measurements need to be performed at different places around 
the joint for multiple subjects in order to match the natural skin deformation during flexion.

\section{Conclusion}

Full-field measurements using fringe projection combined with digital image correlation was used successfully to characterize displacements, deformations and slippage at the brace/skin boundary during knee flexion. Results showed an important migration of the anterior part of the thigh, combined with large strain magnitudes in the skin, twice as much as in the brace. Brace slippage was moderate and turned out to be mostly elastic, i.e. almost no irreversible migration was observed after several flexion-extension cycles. It was assumed that the adverse effects of the brace - slippage and skin pulling - were caused by a critical mismatch in mechanical behaviour between fabric and skin.

A compromise between patient-specific designs and manufacturing costs was proposed based on mechanical optimization of the fabric to match skin behaviour, which was shown to be significantly different between genders: anatomical concerns should not be the only factor to consider to design comfortable braces and improve compliance to these devices.

There are many areas for further development using this quick and non-invasive procedure. For instance, a more systematic study of various areas around the joint to characterize local skin deformations may offer a solid basis to be used for optimizing orthotic devices, surgery planning and other applications. 
Acknowledgements This work was funded in part by the ANRT (Association Nationale de la Recherche et de la Technologie) and the following orthotic manufacturers: Thuasne ${ }^{\circledR}$, Gibaud ${ }^{\circledR}$ and Lohmann-Rauscher ${ }^{\circledR}$.

\section{References}

1. Agache PG, Monneur C, Leveque JL, Rigal JD (1980) Mechanical properties and young's modulus of human skin in vivo. Archives of Dermatological Research 269(3):221-232, DOI 10.1007/BF00406415

2. Avril S, Feissel P, Pierron F, Villon P (2008) Estimation of the strain field from full-field displacement noisy data: comparing finite elements global least squares and polynomial diffuse approximation. European Journal of Computational Mechanics/Revue Européenne de Mécanique Numérique 17(5-7):857868

3. Badulescu C, Bornert M, Dupré JC, Equis S, Grédiac M, Molimard J, Picart P, R R (2013) Demodulation of spatial carrier images: Performance analysis of several algorithms. Experimental Mechanics 53:1357-1370, DOI 10.1007/ s11340-013-9741-6

4. Berge-Gras R, Molimard J (2009) Optimisation de la dérivation en fonction de la résolution et de la résolution spatiale sur les déformations. In: Actes du XIXème Congrès Français de Mécanique, Marseille, France

5. Bethke K (2005) The second skin approach : skin strain field analysis and mechanical counter pressure prototyping for advanced spacesuit design. Thesis, Massachusetts Institute of Technology

6. Breque C, Dupre JC, Bremand F (2004) Calibration of a system of projection moiré for relief measuring: biomechanical applications. 
Optics and Lasers in Engineering 41(2):241-260, DOI http://dx.doi. org/10.1016/S0143-8166(02)00198-7, URL http://www.sciencedirect.com/ science/article/pii/S0143816602001987

7. Brownstein B (1998) Migration and design characteristics of functional knee braces. Journal of Sport Rehabilitation 7(1):33-43

8. Chew KTL, Lew HL, Date E, Fredericson M (2007) Current evidence and clinical applications of therapeutic knee braces. American Journal of Physical Medicine \& Rehabilitation 86(8):678-686, DOI 10.1097/PHM. 0b013e318114e416

9. CMV AG (2014) Contrex online documentation. http://www.con-trex. ch/index $\cdot$ php?option=com_content\&task=blogcategory\&id=15\&Itemid=82, [Online; accessed 21-March-2014]

10. Cua AB, Wilhelm KP, Maibach HI (1990) Elastic properties of human skin: relation to age, sex, and anatomical region. Archives of Dermatological Research 282(5):283-288, DOI 10.1007/BF00375720

11. Diridollou S, Black D, Lagarde J, Gall Y, Berson M, Vabre V, Patat F, Vaillant L (2000) Sex- and site-dependent variations in the thickness and mechanical properties of human skin in vivo. International Journal of Cosmetic Science 22(6):421-435, DOI 10.1111/j.1468-2494.2000.00037.x

12. Evans SL, Holt CA (2009) Measuring the mechanical properties of human skin in vivo using digital image correlation and finite element modelling. The Journal of Strain Analysis for Engineering Design 44(5):337-345, DOI 10. 1243/03093247JSA488

13. Guimberteau J, Sentucq-Rigall J, Panconi B, Boileau R, Mouton P, Bakhach J (2005) Introduction à la connaissance du glissement des structures sous- 
cutanées humaines. Annales de Chirurgie Plastique Esthétique 50(1):19-34, DOI 10.1016/j.anplas.2004.10.012

14. iData Research (2012) U.S. market for orthopedic braces \& support devices 2012. Tech. rep.

15. Institut Français du Textile et de l'Habillement (2006) Campagne nationale de mensuration

16. Molimard J, Navarro L (2013) Uncertainty on fringe projection technique: a Monte-Carlo-based approach. Optics and Laser in Engineering 51(7):840-847

17. Molimard J, Boyer G, Zahouani H (2010) Frequency-based image analysis of random patterns: an alternative way to classical stereocorrelation. Journal of the Korean Society for Nondestructive Testing 30(3):181-193

18. Ounpuu S (1994) The biomechanics of walking and running. Clinics in sports medicine 13(4):843-863

19. Pierrat B, Molimard J, Navarro L, Avril S, Calmels P (2013) Evaluation of the mechanical efficiency of knee braces based on computational modeling, DOI $10.1080 / 10255842.2013 .832227$

20. Pierrat B, Molimard J, Navarro L, Avril S, Calmels P (2014) Evaluation of the mechanical efficiency of knee orthoses: a combined experimentalnumerical approach. Proceedings of the Institution of Mechanical Engineers, Part H: Journal of Engineering in Medicine 228(6):533-546, DOI 10.1177/ 0954411914533944

21. Regalbuto MA, Rovick JS, Walker PS (1989) The forces in a knee brace as a function of hinge design and placement. The American journal of sports medicine 17(4):535-543 
22. Singer J, Lamontagne M (2008) The effect of functional knee brace design and hinge misalignment on lower limb joint mechanics. Clinical biomechanics 23(1):52-59, DOI 10.1016/j.clinbiomech.2007.08.013

23. Thoumie P, Sautreuil P, Mevellec E (2001) Orthèses de genou. première partie : Évaluation des propriétés physiologiques à partir d'une revue de la littérature. knee orthosis. first part: evaluation of physiological justifications from a literature review. Annales de Réadaptation et de Médecine Physique 44(9):567-580, DOI 10.1016/S0168-6054(01)00157-X

24. Thoumie P, Sautreuil P, Mevellec E (2002) Orthèses de genou. Évaluation de l'efficacité clinique à partir d'une revue de la littérature. Annales de Réadaptation et de Médecine Physique 45(1):1-11, DOI 10.1016/S0168-6054(01)00166-0

25. Van Leerdam N (2006) The genux, a new knee brace with an innovative nonslip system. In: Academy of American Orthotists and Prosthetists, Chicago, IL 